\title{
MRT nach Patellaluxation: Quantifizierung der Risiko- faktoren und Beschreibung der Folgeschäden
}

\author{
MRI after Patellar Dislocation: Assessment of Risk Factors and Injury to the Joint
}

\author{
Autoren \\ G. Diederichs ${ }^{1}$, S. Scheffler ${ }^{2,3}$ \\ Institute \\ Radiologie, Charité - Universitätsmedizin Berlin, Campus Charité Mitte \\ 2 Zentrum für Muskuloskeletale Chirurgie, Charité - Universitätsmedizin Berlin, Campus Charité Mitte \\ Chirurgisch Orthopädischer PraxisVerbund, Berlin
}

Key words
knee
patella
dislocation
MRI

eingereicht 11.7.2012

akzeptiert $\quad 15.1 .2013$

Bibliografie

DOI http://dx.doi.org/

10.1055/s-0032-1330690

Online-Publikation: 14.3.2013

Fortschr Röntgenstr 2013; 185:

611-620 @ Georg Thieme

Verlag KG Stuttgart · New York ·

ISSN 1438-9029

\section{Korrespondenzadresse}

Dr. Gerd Diederichs

Radiologie, Charité -

Universitätsmedizin Berlin,

Campus Charité Mitte

Charitéplatz 1

10117 Berlin

Tel.: ++49/30/450627094

Fax: ++49/30/450527911

gerd.diederichs@charite.de

\section{Zusammenfassung}

$\nabla$

Bei der Patellaluxation handelt es sich um ein Herausspringen der Kniescheibe aus dem trochlearen Gleitlager nach lateral. In der Regel liegen anatomische Risikofaktoren in unterschiedlichem Ausprägungsgrad vor, die in Zusammenhang mit einer Rotationsbewegung des Beines zu einem Luxationsereignis führen. Die im MRT problemlos diagnostizierbaren Kontusionsödeme der inferomedialen Patella und des lateralen Femurkondylus sowie die Ruptur des medialen patellofemoralen Ligamentes gelten als typische Zeichen einer kürzlich stattgehabten Luxation. Allerdings sind auch osteochondrale Begleitverletzungen möglich, die je nach Ausmaß eine Indikation zur frühen Refixation darstellen können. Nach dem Erstereignis folgen durch den instabilen Kapsel-BandApparat häufig weitere Luxationen, die in Abhängigkeit der vorliegenden anatomischen Normvarianten bis hin zu einer chronischen patellofemoralen Instabilität führen können. Therapeutisch hat sich ein breites Spektrum aus konservativen und operativen Verfahren durchgesetzt. Während Erstluxationen häufig konservativ behandelt werden, orientiert sich das operative Verfahren nach der Zweitluxation am Verletzungsmuster und Ausprägungsgrad der anatomischen Risikofaktoren. Die relevanten prädisponierenden Anlagevarianten sind die Trochleadysplasie, die Patella alta und der pathologische Abstand der Tuberositas tibia zum Sulcus trochleae (TT-TG). Insgesamt sollten im radiologischen Befund sowohl die Folgeschäden der Luxation als auch die anatomischen Risikofaktoren quantifiziert werden, da eine individuelle Analyse des zugrunde liegenden Pathomechanismus ausschlaggebend für die optimale patientenspezifische Therapie ist.

\section{Abstract \\ $\nabla$}

Patellar dislocation is the lateral displacement of the patella from the femoral trochlea. Affected individuals typically have underlying anatomic risk factors of variable magnitude, which, in conjunction with leg rotation, cause the event. Magnetic resonance imaging (MRI) permits straightforward diagnosis of the typical features of recent patellar dislocation: contusion edema of the inferomedial patella and the lateral femoral condyle as well as rupture of the medial patellofemoral ligament. In case of concomitant osteochondral injury, early surgical refixation may be indicated, depending on the size. After a first dislocation, which can damage the capsuloligamentous stabilizers, subjects may sustain further dislocations or even develop chronic patellofemoral instability, depending on the presence and severity of anatomic variants. A wide range of conservative and surgical treatments are available. While a first patellar dislocation is often treated conservatively, surgical strategies after a second dislocation depend on the pattern of injury and the severity of underlying anatomic risk factors. The most relevant predisposing variants are trochlear dysplasia, patella alta, and an abnormal tibial tubercle to trochlear groove distance (TT-TG). The radiologist's report should give a quantitative estimate of both the injuries resulting from dislocation and the underlying anatomic risk factors. An accurate characterization of the individual pathomechanism is crucial for tailoring treatment. 


\section{Terminologie}

$\nabla$

Die Luxation der Patella bedeutet ein Herausgleiten der Kniescheibe aus der trochlearen Führung und ist durch einen vollständigen Kontaktverlust der patellofemoralen Gelenkflächen definiert [1]. Eine spontane Reposition kann unmittelbar nach dem Ereignis durch Streckung des Beines erfolgen oder bei fixierter Luxation durch manuelle Verfahren im Rahmen der Erstbehandlung erreicht werden. Bei der Subluxation der Patella ist die Kniescheibe nicht mehr im Sulkus der Trochlea zentriert, sondern lateralisiert und überragt mit ihrer Außenkante deutlich die Trochleafacette. Die patellofemorale Instabilität ist der allgemeine Oberbegriff für Patellaluxation und -subluxation. Die akute Patellaluxation bezeichnet meist das Erstereignis, wobei eine Zweitluxation oder weitere Ereignisse als rezidivierende Luxationen bezeichnet werden [2]. Eine chronische Luxation oder chronische patellofemorale Instabilität ist nicht klar definiert, bezeichnet aber in der Regel permanentes Maltracking mit Subluxationen bis hin zu permanenter Luxationsstellung [1, 3].

Am häufigsten liegen habituelle Luxationen vor, bei denen zugrunde liegende Anlagevarianten die Luxation begünstigen und meist lediglich ein Bagatelltrauma zum Herausspringen der Kniescheibe führt [1, 2]. Bei den rein traumatischen Luxationen wird das Herausspringen primär durch eine signifikante Krafteinwirkung erklärt (z.B. direkter Schlag gegen die Patella), weswegen in solchen Fällen keine anatomischen Risikofaktoren vorliegen müssen. In der MR-Diagnostik kaum vertretene Varianten sind die kongenitale oder neurogene Patellaluxation.

\section{Epidemiologie \\ $\nabla$}

Die Inzidenz von akuten Luxationen der Patella wird je nach Studie mit 6- 77 pro 100000 angegeben [4 - 7]. Das Durchschnittsalter liegt bei ungefähr 20 Jahren [8]. Häufig sind junge und aktive Frauen betroffen, wobei zwei Drittel der Erstluxationen beim Sport stattfinden [5]. Insbesondere im Jugendalter weisen Frauen ein um ein Drittel höheres Risiko für Patellaluxationen auf als Männer [5]. Die erstmalige Luxation kann bei vielen Patienten beträchtliche Konsequenzen nach sich ziehen, nicht nur durch die resultierenden Kniebinnenschäden, sondern auch durch weitere Luxationen. In der Erholungsphase ist die Beweglichkeit deutlich eingeschränkt und ein anteriorer Knieschmerz kann bestehen bleiben [4, 9]. Etwa die Hälfte der Patienten wird ein zweites Luxationsergeignis erleiden, da der Kapsel-Band-Apparat insuffizient ist $[5,10]$. Konsekutive, rezidivierende Luxationen und eine chronische patellofemorale Instabilität prädisponieren zu früher patellofemoraler Arthrose, wodurch die Lebensqualität erheblich eingeschränkt sein kann [11 -13].

\section{Ätiologie}

Die Entstehung einer patellofemoralen Instabilität ist nicht vollständig geklärt und wird trotz zahlreicher Publikationen nach wie vor kontrovers diskutiert. Im Normalfall wird das Patellofemoralgelenk durch statische (Trochlea), aktive (Muskeln, z.B.M. vastus medialis obliquus) und passive (Bänder, z.B. Retinakula) Stabilisatoren geführt, wodurch die Patella während Kniebeugung regelrecht mit dem ossären Gleitlager der Trochlea artikuliert. Die Erstluxation findet beim überwiegenden Teil der Patienten im Rahmen von sportlichen Aktivitäten durch Innenrotation des am
Boden fixierten Beines und entsprechenden Valgusstress des Kniegelenks statt. Durch einen resultierenden Kraftvektor nach lateral springt die Patella aus dem Gleitlager und prallt gegen die Außenseite des lateralen Femurkondylus. Häufig zerreißt dabei das mediale patellofemorale Ligament (MPFL), das einen Teil des medialen Retinakulum darstellt und ein ausschlaggebender ligamentärer Stabilisator der Patella ist. Bei inadäquater Behandlung des rupturierten Bandes kann das MPFL narbig heilen, ohne die initial stabilisierende Funktion wiedererlangen zu können, wodurch eine chronische patellofemorale Instabilität begünstigt wird.

\section{Risikofaktoren \\ $\nabla$}

Es sind unterschiedliche anatomische Risikofaktoren bekannt, die eine patellofemorale Instabilität begünstigen können und die Wahrscheinlichkeit von Folgeluxationen erhöhen. $\mathrm{Zu}$ den wichtigsten Risikofaktoren zählen die Trochleadysplasie, die Patella alta und ein pathologischer TT-TG-Abstand, wobei ein breites Ausprägungsspektrum dieser Varianten besteht [14]. Dies reicht vom Fehlen jeglicher Risikofaktoren bis zum Vorliegen von einem oder auch mehreren Risikofaktoren [15]. Es ist daher sinnvoll, diese 3 anatomischen Parameter im radiologischen Befund zu quantifizieren, da ihr Vorhandensein direkten Einfluss auf die Wahl der optimalen Therapie hat ( $\bullet$ Tab. 1).

\section{Trochleadysplasie}

Die Trochleadysplasie gilt als eine der wichtigsten anatomischen Anlagestörungen, die eine patellofemorale Instabilität begünstigen [16 - 22]. Da sie in bis zu 93 \% der Fälle bilateral auftritt, gehen Dejour et al. von einer konstitutionellen Abnormalität aus [18]. Insgesamt ist die trochleare Gelenkfläche abgeflacht, sodass vor allem in strecknaher Position kein zentriertes Eintreten der Patella in den Sulcus trochlearis erfolgen kann und eine sichere ossäre Stabilisierung des Gleitvorgangs nicht mehr gewährleistet

\begin{tabular}{|c|c|}
\hline \multicolumn{2}{|c|}{ Risikofaktoren } \\
\hline $\begin{array}{l}\text { Trochlear- } \\
\text { dysplasie }\end{array}$ & $\begin{array}{l}\text { morphologische Einteilung Dejour-Klassifikation } \\
\text { (A-D) ( } \bullet \text { Abb. 1) }\end{array}$ \\
\hline & $\begin{array}{l}\text { quantitative Bestimmung }(\text { Abb. } 2 \text { ): } \\
\text { - Trochleartiefe Grenzwert }<3 \text { mm } \\
\text { - Trochlear-Facetten-Asymmetrie-Grenzwert } \\
\quad<40 \% \\
\text { - Laterale-Trochlear-Inklination-Grenzwert }<11^{\circ}\end{array}$ \\
\hline Patella alta & $\begin{array}{l}\text { Insall-Salvati-Index Grenzwert > 1,3 ( } \bullet \text { Abb. } 3 \text { ) } \\
\text { Canton-Duchamps-Index Grenzwert > 1,2 }\end{array}$ \\
\hline TT-TG & $\begin{array}{l}\text { normal bis } 15 \mathrm{~mm}(\mathbf{A b b} .4) \\
\text { grenzwertig } 15-20 \mathrm{~mm} \\
\text { pathologisch > } 20 \mathrm{~mm}\end{array}$ \\
\hline \multicolumn{2}{|c|}{ Folgeschäden } \\
\hline+++ & $\begin{array}{l}\text { Kontusionsödeme der medialen Patella und des } \\
\text { lateralen Femurkondylus }\end{array}$ \\
\hline+++ & Ruptur oder Teilruptur des MPFL \\
\hline+++ & osteochondrale Fraktur der inferomedialen Patella \\
\hline++ & $\begin{array}{l}\text { osteochondrale Fraktur des lateralen Femur- } \\
\text { kondylus }\end{array}$ \\
\hline++ & Knorpelschaden der zentralen Patella \\
\hline+ & $\begin{array}{l}\text { Knorpelschaden der lateralen Patella und der } \\
\text { Trochlea }\end{array}$ \\
\hline+ & Patellofemoralarthrose \\
\hline
\end{tabular}



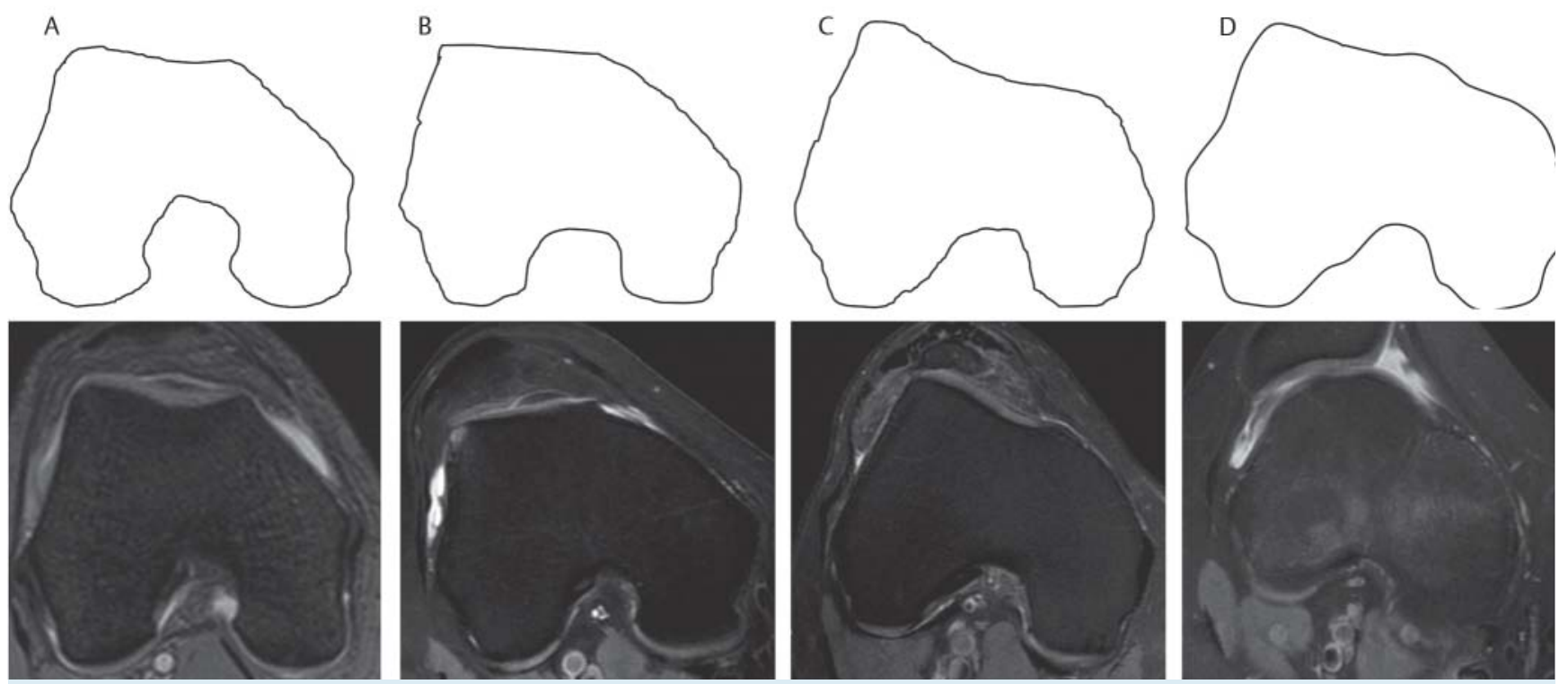

Abb. 1 Einteilung der Trochleadysplasie nach Dejour in 4 Grade A-D. Typ A: Verminderte Sulkustiefe. Typ B: Horizontale Trochlea. Typ C: Asymmetrie der Facetten, ansteigender lateraler Fenurkondylus. Typ D: Typ B + C und buckelartige Vorwölbung der zentralen Trochlea.

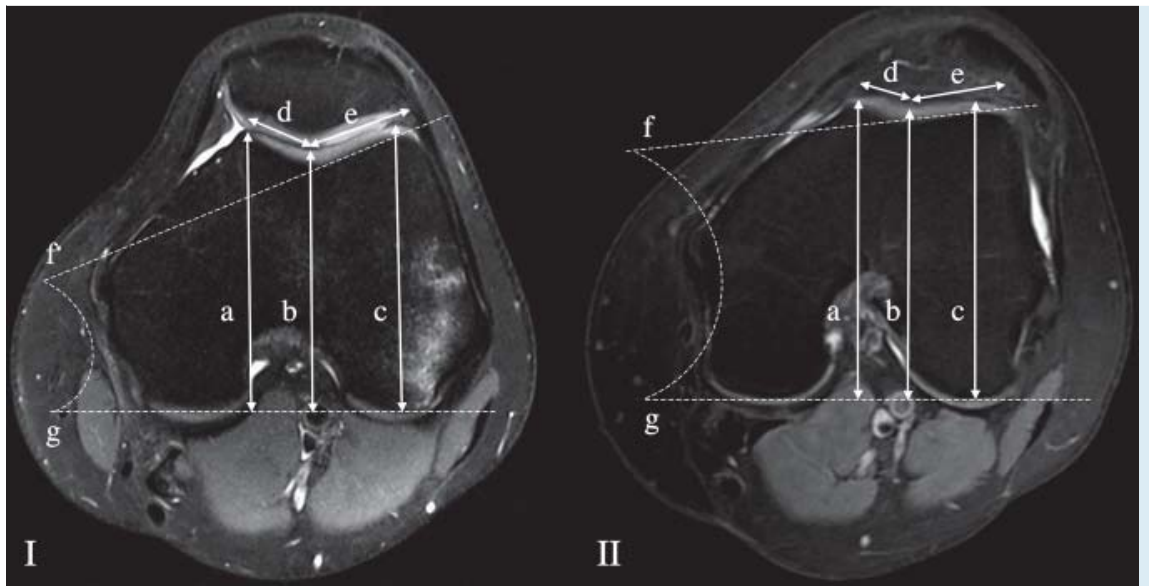

Abb. 2 Messparameter Trochleadysplasie. Vermessung von 3 Parametern zur Evaluation der Trochleadysplasie bei einem Patienten ohne Dysplasie (I) und einem Patienten mit Dysplasie (II). Trochleartiefe vom Mittelwert der Abstände der medialen (a) und lateralen (c) Trochleafacette zur dorsalen Femurkondylenebene $(\mathrm{g})$ wird der Abstand des Sulcus (b) subtrahiert $=(a+c) / 2-b$. Grenzwert $\leq 3 \mathrm{~mm}$. Trochlear-Facetten-Asymmetrie-Verhältnis der Länge der medialen Trochleafacette (d) geteilt durch die Länge der lateralen Trochleafacette (e) in Prozent $=\mathrm{d} / \mathrm{e}-100 \%$. Grenzwert $\leq 40 \%$ Laterale-Trochlear-Inklination-Winkel zwischen einer Linie entlang des subchondralen Knochens der lateralen Trochlea ( $f$ ) und der dorsalen Femurkondylenebene $(\mathrm{g})$. Grenzwert $<11^{\circ}$.

ist. Dadurch lateralisiert die Patella bereits bei Beginn der Flexion und eine Luxation wird begünstigt. Bei hochgradiger Dysplasie kann die Trochlea vollkommen horizontal ohne Vertiefung oder gar nach außen konvex konfiguriert sein.

Klassische Zeichen der Trochleadysplasie in axial aquirierten Sulkus-Projektionsaufnahmen bestehen bei fast allen Luxationspatienten: eine Abflachung der Trochlea und ein hoher Sulkuswinkel $>145^{\circ}$ sowie der an der proximalen Trochlea zu beobachtende Buckel und das „crossing sign“, das durch eine Überlagerung der unterschiedlich hohen medial und lateralen Trochleakanten im Seitbild entsteht [18]. In der klinischen Praxis hat sich insbesondere in unfallchirurgischen/orthopädischen Abteilungen die qualitative Einteilung der Trochleadysplasie in 4 Gruppen nach der Klassifikation von Dejour durchgesetzt [23]. Die ursprünglich anhand von konventionellen Aufnahmen vorgenommene Klassifikation ist auch auf axiale MRT-Bilder übertragbar [24] (• Abb. 1). Bezüglich quantitativer Messmethoden zur Diagnostik der Trochleadysplasie kann in axialen MR-Bildern mit hoher Genauigkeit die Einstellung des Gelenkknorpels des femoralen Sulkus als eigentlich relevante Gelenkfläche vermessen werden [14, 21].
In einer Vielzahl von MRT-basierten Studien wurde die Trochleadysplasie von Luxationspatienten ausgewertet [16, 17, 19-22, 25, 26]. Empfehlenswerte, standardisierte Messparameter mit hoher diagnostischer Genauigkeit sind die Trochleatiefe und die Trochlear-Facetten-Asymmetrie (beide Sensitivität 100\%, Spezifität 96\%) [21] ( $\triangle$ Abb.2). Für beide Messtechniken wird eine axiale Schicht ca. $3 \mathrm{~cm}$ oberhalb des Gelenkspalts ausgewählt, auf der trochlearer Gelenkknorpel abgebildet sein muss. Je nach Länge der Trochlea und Schichtdicke muss gegebenenfalls eine benachbart gelegene Schicht verwendet werden, generell sollte die erste Schicht gewählt werden, auf der die Trochlea mit Knorpel bedeckt ist. Für die Trochleatiefe wird an der Oberfläche des Knorpels der tiefste Punkt des Sulkus vermessen. Messwerte von $3 \mathrm{~mm}$ oder weniger bedeuten eine Dysplasie. Für die TrochlearFacetten-Asymmetrie wird die Ratio aus Breite der medialen und lateralen Trochleafacette vermessen - bei einer Ratio von $<40 \%$ liegt eine Trochleadysplasie vor. Des Weiteren kann noch die Laterale-Trochlear-Inklination bestimmt werden. Sie ist als der Winkel zwischen dem subchondralen Knochen der lateralen Trochleafacette und einer Linie entlang der Rückfläche der Femurkondylen definiert ( $\bullet$ Abb. 2). Der Grenzwert für eine Troch- 
leadysplasie ist $<11^{\circ}$ (Sensitivität $93 \%$, Spezifität 87\%) [17]. Bei hochgradiger Dysplasie ist kein Sulcus trochlearis ausgebildet, wodurch die Vermessung der Parameter erschwert werden kann. Insbesondere in solchen Fällen ist für den radiologischen Befund eine morphologische Einteilung anhand der Dejour-Klassifikation sinnvoll. Vor allem geringgradige Dysplasien können auch bei Individuen ohne primäre patellofemorale Instabilität vorkommen [16, 19-21]. Kommt es jedoch durch ein Trauma zu einer Luxation, bieten die Bänder keine ausreichende Stabilisierung mehr, wodurch auch unter diesen Umständen eine chronische Instabilität resultieren kann.

\section{Patella alta}

Die hochstehende Patella ist ein weiterer anatomischer Risikofaktor, der mit rezidivierenden Patellaluxationen in Zusammenhang gebracht wird [15, 16, 18-20, 27 -30]. Im Normalfall befindet sich die Patella beim gestreckten Bein oberhalb der Trochlea und wird erst bei zunehmender Beugung durch das Trochleazentrum gesichert [31]. Ist der Abstand pathologisch vergrößert, so folgt eine verspätete Artikulation der Gelenkflächen und dadurch verminderte ossäre Führung. Je nach Studie weist bis zu der Hälfte der Patienten nach akuter Patellaluxation im MRT eine Patella alta auf, wobei aber auch bei 10 - $23 \%$ von Normalkollektiven pathologische Werte gemessen werden $[15,16,19]$.

Es sind zahlreiche Methoden zur Vermessung des Abstands der Patella zum Tibiaplateau bekannt, die jeweils spezifische Vorund Nachteile aufweisen. In der klinischen Praxis haben sich für die Auswertung an sagittalen MRT-Bildern der Insall-Salvati-Index und der Canton-Duchamps-Index etabliert, die jeweils eine robuste Reproduzierbarkeit aufweisen [32, 33]. Die ursprünglich an konventionellen Seitbildern entwickelten Messtechniken sind mit hoher Übereinstimmung an sagittalen MR-Bildern anwendbar [29, 34].

Für die Bestimmung des Insall-Salvati-Index wird der Längsdurchmesser der Patella zur Länge der Patellasehne ins Verhältnis gesetzt ( $\bullet$ Abb. 3) [35]. Bei der Abmessung an Röntgenbildern wurde ursprünglich ein Grenzwert von $\geq 1,2$ für das Vorliegen eines Patellahochstands angegeben $[35,36]$. In einer Studie von Miller et al. wurde die Übertragbarkeit des Messverfahrens auf sagittale MRT-Bilder getestet und ein etwas höherer Grenzwert von $\geq 1,3$ empfohlen [34], der auch in aktuellen Studien an Luxationspatienten angewendet wurde [16, 19, 34].

Der Canton-Duchamps-Index berechnet sich als Quotient aus der Länge der Retropatellarfläche und dem Abstand der unteren Patellakante zur Vorderkante des Tibiaplateaus ( $\bullet$ Abb. 3) [37]. Hier gilt ein Grenzwert von $\geq 1,2$ für das Vorliegen einer Patella alta; dieser Grenzwert kann auch bei Kindern verwendet werden $[18,37,38]$.

\section{TT-TG-Abstand}

Ist die Tuberositas tibiae als Ansatzpunkt der Patellasehne im Vergleich zum Sulkus der Trochlea lateralisiert, so besteht ein Kraftvektor nach außen, wodurch eine patellofemorale Instabilität begünstigt wird. Mehrere Studien haben gezeigt, dass ein pathologischer TT-TG-Abstand einen signifikanten Risikofaktor für Patellaluxationen darstellt $[16,18,25,39,40]$ und bei über der Hälfte der Luxationspatienten vorhanden ist [18]. Allerdings sollte bemerkt werden, dass ein pathologischer TT-TG nur sehr selten als alleiniger anatomischer Risikofaktor vorliegt und in der Regel mit einer Trochleadysplasie vergesellschaftet ist [15]. Obwohl die CT als Goldstandard für die TT-TG-Vermessung angesehen wird, kann die Distanz ebenfalls im MRT bestimmt werden, eine CT braucht nicht zusätzlich durchgeführt zu werden [41].

Ziel der Messung ist in der obersten axialen Schicht auf der erstmalig komplett eine vollständige knorpelige Deckung der Trochlea vorliegt, zunächst den tiefsten Punkt der Trochleagrube zu identifizieren und dann durch diesen Punkt rechtwinklig zur Dorsalebene der Femurkondylen eine Linie zu zeichnen $(\bullet$ Abb.4). Eine zweite Linie wird parallel durch den Mittelpunkt der Tuberositas gezogen. Der TT-TG-Abstand ergibt sich aus der Distanz zwischen den beiden Linien. Es ist allerdings zu beachten, dass bei ausgeprägter Trochleadysplasie keine vermessbare Vertiefung der Trochlea ausgebildet sein kann, weswegen in diesen Fällen der TT-TG nicht bestimmbar ist.

Bei Kontrollgruppen ohne patellofemorale Instabilität sind TTTG-Mittelwerte von 10-12 mm publiziert [25, 39, 42]. Werte zwischen $15-20 \mathrm{~mm}$ werden als grenzwertig angesehen. Messwerte von $>20 \mathrm{~mm}$ werden als definitiv pathologisch eingestuft und können durch operativen Versatz der Tuberositas korrigiert werden [43].

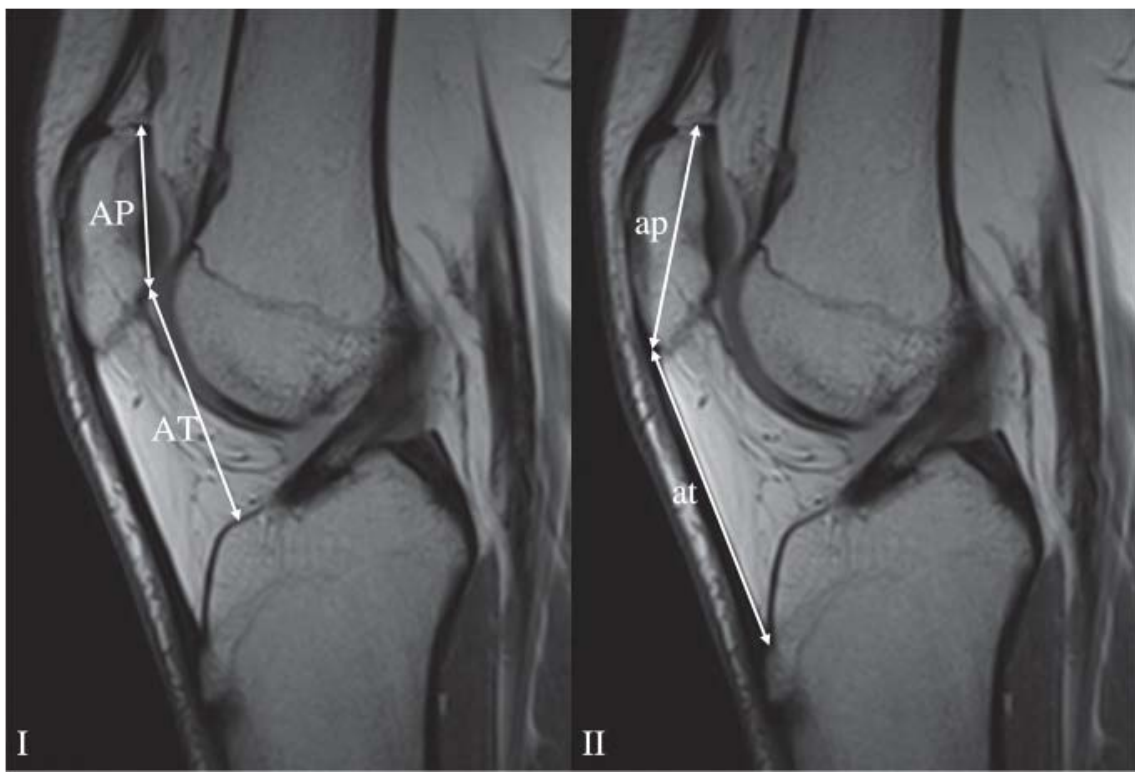

Abb. 3 Messparameter Patella alta. I) Messung des Patellar Index nach Caton-Duschamps: AT/AP. Bei einem Index von $\geq 1,2$ besteht eine Patella alta. II) Messung des Patellar-Index nach Insall-Salvati: at/ap. Bei einem Index $\geq 1,3$ besteht eine Patella alta. Bei dieser Patientin lag ein Canton-DuschampsIndex von 1,55 und ein Insall-Salvati-Index von 1,45 vor. 


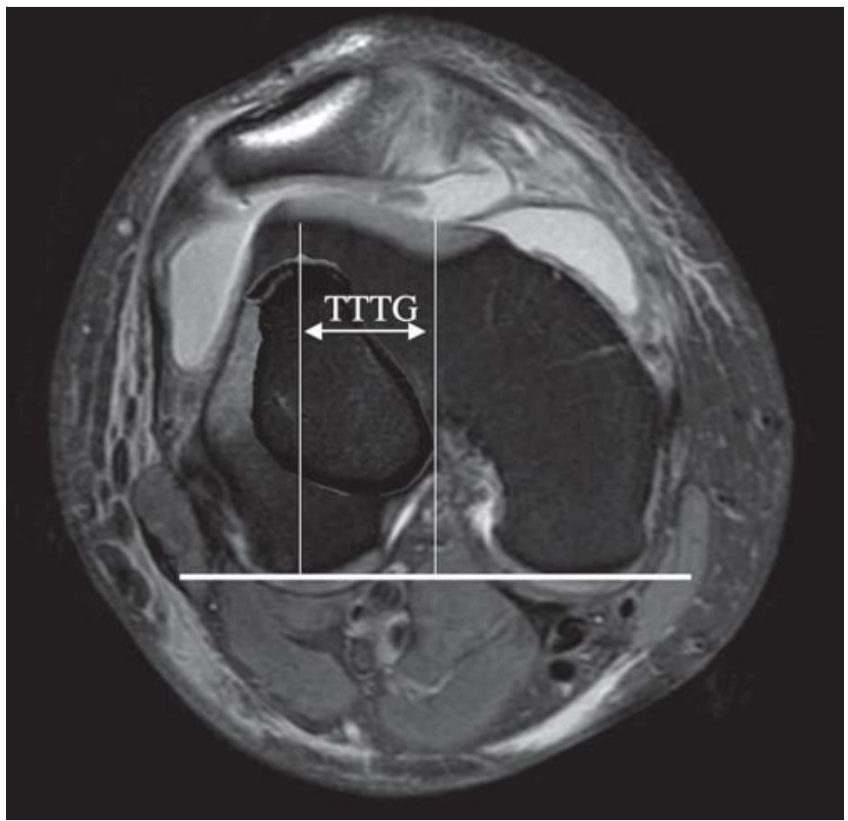

Abb.4 TTTG. Tibial-Tuberosity-to-Trochlear-Groove-Entfernung: Es wird zunächst die erste axiale Schicht ausgewählt, auf der die Trochlea vollständig mit Knorpel bedeckt ist. Hier wird der Abstand einer Linie durch den tiefsten Punkt der Trochlea, die rechtwinklig zur dorsalen Femurkondylenebene ausgerichtet ist, zu einer Linie durch die Mitte der Tuberositas gemessen (hier in das Bild kopiert). Messwerte von $\geq 20 \mathrm{~mm}$ bedeuten einen pathologischen TT-TG-Abstand.

\section{Weitere}

Ob die Rotationseinstellung des Beines (femorale Anteversion, tibiale Außenrotation) und die mechanische Tragachse (Genu valgum/varum) als valide Risikofaktoren für patellofemorale Instabilität angesehen werden können, ist noch nicht eindeutig geklärt. Eine aktuelle Studie hat gezeigt, dass Luxationspatienten im Vergleich zur Kontrollgruppe eine im Mittel höhere femorale Anteversion und eine Tendenz zum genu valgum aufweisen [44]. Weitere ältere Studien zeigen einen Zusammenhang der femoralen oder tibialen Torsion zur Instabilität [45 - 47], während andere Studien keinen Einfluss darstellen können, insbesondere da die Varianz in Normalkollektiven zu stark variiert, um pathologische Grenzwerte festlegen zu können [18, 48]. Bei klinischem Verdacht auf Alignmentstörung können mittels schneller axialer T1w- oder T2w-Sequenzen die Gelenke der Beine innerhalb weniger Minuten abgebildet und die relevanten Achsen vermessen werden. Nach orthopädischer Literatur können bei Vorliegen von Extremwerten in Einzelfällen femorale oder tibiale Derotationsosteotomien indiziert sein [49]. Der Einfluss der Patellaform als primärer Risikofaktor für patellofemorale Instabilität ist ebenfalls unsicher und hat sich in der aktuellen Diagnostik der patellofemoralen Instabilität nicht durchgesetzt, weswegen die Wiberg-Klassifikation in der heutigen patientenspezifischen Erstellung des Risikofaktorprofils keine breite Akzeptanz mehr findet. Auch bei dem von Dejour beschriebenen Patella-Tilt, der eine Verkippung der Patellaquerachse nach lateral darstellt, scheint es sich eher um eine Folgeerscheinung unterschiedlicher anderer Faktoren (rupturiertes oder insuffizientes MPFL, Patella alta, Trochleadysplasie) zu handeln, als um eine tatsächliche Ursache für patellofemorale Instabilität [50]. Abgesehen von knöchernen Anlagevarianten wird außerdem eine allgemeine ligamentäre Hyperlaxität bei Luxationspatienten diskutiert [51, 52], was jedoch von einer anderen Studie nicht bestä-

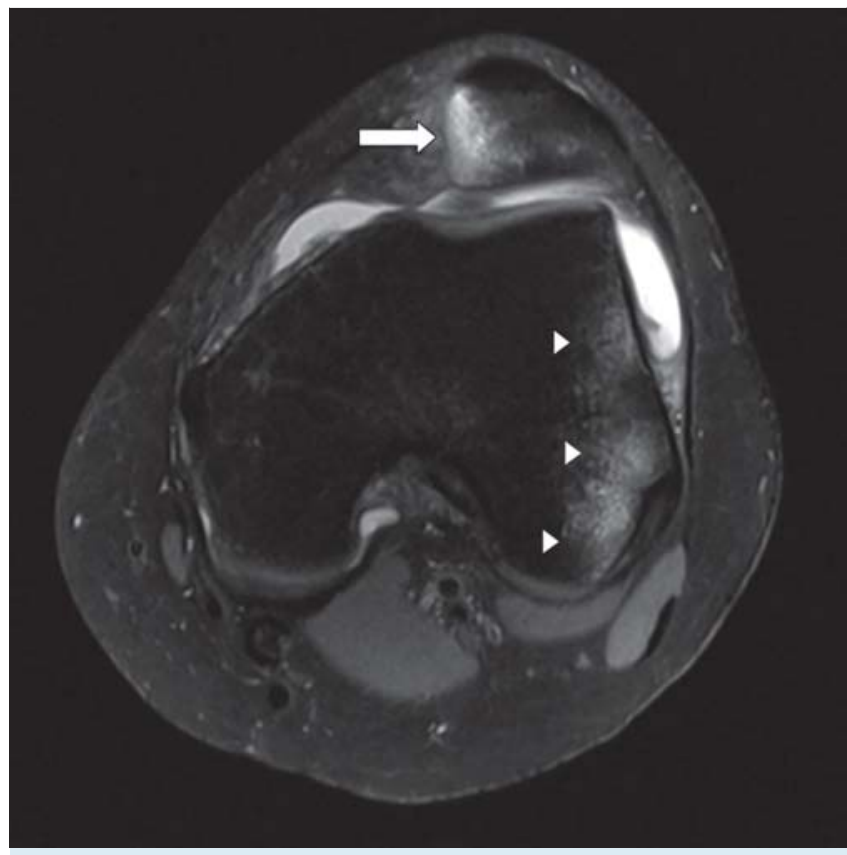

Abb.5 Kontusionsödeme. 20-jährige Patientin unmittelbar nach Erstluxation der Patella. Typische Knochenödeme an der inferomedialen Patella (Pfeil) und an der Außenfläche des lateralen Femurkondylus (Pfeilspitzen).

tigt werden konnte [4]. Zumindest bei rezidivierenden Luxationen scheint die generelle Hypermobilität eine Rolle zu spielen [53]. Auch ob eine Hypotrophie des Vastus medialis obliquus initial zu einer Erstluxation beiträgt oder erst als dessen Folge entsteht und dann eine chronische patellofemorale Instabilität begünstigt, ist nicht eindeutig geklärt.

\section{Folgeschäden}

Kürzlich stattgehabte Luxationen der Patella zeigen ein typisches Befundmuster und sind im MRT einfach zu diagnostizieren. Anschlagsbedingte Knochenödeme der inferomedialen Patella und der Außenseite des lateralen Femurkondylus sowie eine Ruptur des MPFL gelten als pathognomonisch für eine stattgehabte laterale Patellaluxation [19, 54 -57]. Außerdem können osteochondrale oder chondrale Verletzungen direkte Folge des Luxationsereignisses sein. Insbesondere bei chronischer patellofemoraler Instabilität droht eine frühe patellofemorale Arthrose [58] ( $\bullet$ Tab. 1).

\section{Osteochondrale und chondrale Verletzungen}

Die mediale Patella weist durch die Kontusion gegen das Femur bei fast allen Patienten unmittelbar nach Erstluxation ein Knochenödem auf ( $\bullet$ Abb.5). Bei 70\% der Patienten besteht an der Konstusionsstelle ein osteochondraler Schaden und bei $40 \%$ wird eine typische konkave Impaktation nachgewiesen [19] ( $\bullet$ Abb.6). Ätiologisch können die primär impaktationsbedingten osteochondralen Schäden der medialen Patella von den avulsionsbedingten Defekten unterschieden werden, die einem ligamentären Ausriss des MPFL zugeordnet werden. Diese frischen Traumafolgen sollten allerdings nicht mit paraossalen Ossifikationen verwechselt werden, die neben der medialen Kante der Patella am Ansatz des MPFL und der Gelenkkapsel anzutreffen 


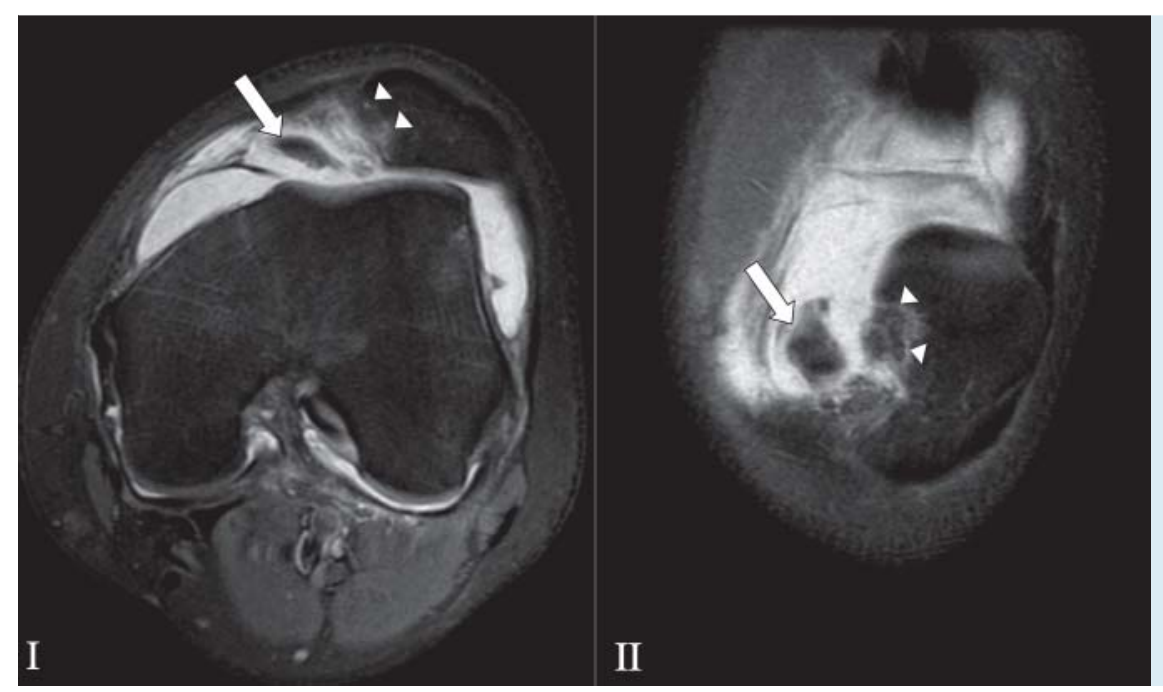

Abb. 6 Osteochondraler Defekt. 21-jähriger Patient unmittelbar nach Erstluxation der Patella. Axiales (I) und koronares (II) Bild eines osteochondralen Defektes der inferomedialen Patella (Pfeilspitzen) mit freiem Gelenkkörper durch disloziertes Fragment (Pfeil).

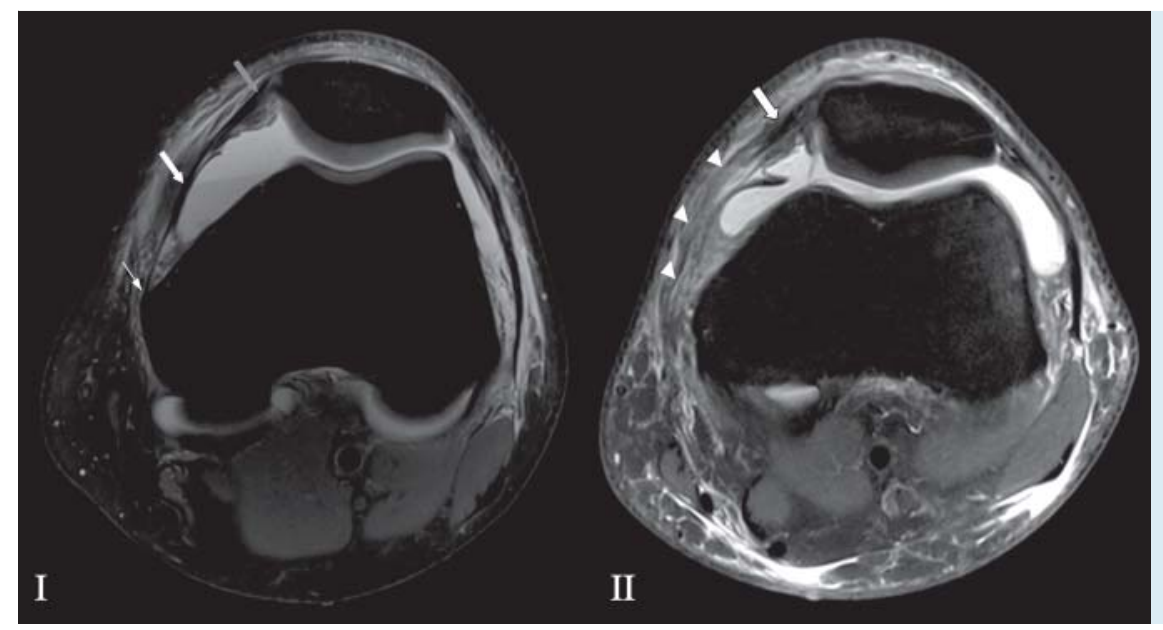

Abb.7 I) Intaktes mediales patellofemorales Ligament (MPFL, dicker weißer Pfeil) bei einem Patienten nach akutem Knietrauma. Das MPFL ist ein horizontal verlaufender Faserzug und entspricht der mittleren Schicht der medialseitigen Kniegelenksfasern. Es entspringt femoralseitig vor dem Epikondylus medialis (dünner weißer Pfeil) und inseriert gemeinsam mit dem medialen Retinakulum an den superioren zwei Dritteln des medialen Patellarands (grauer Pfeil). II) 18-jährige Patientin nach Erstluxation. Vollständige Ruptur des medialen patellofemoralen Ligaments (MPFL) am femoralen Ansatz und im mittleren Drittel (Pfeilspitzen) nach akuter Patellaluxation. Patellare Insertion des MPFL und medialen Retinakulum als Bandstumpf abgrenzbar (Pfeil).

sind und auf repetetiven Weicheilschäden typischerweise nach rezidivierenden Luxationen zurückgeführt werden [59].

Zusätzlich zu den osteochondralen Defekten der medialen Patella sollten Schäden der zentralen Retropatellarfläche ausgeschlossen werden, da frische Knorpeleinrisse oder -abrisse zum Beispiel durch das Gleiten über den lateralen Femurkondylus verursacht werden können. Insbesondere nach rezidivierenden Luxationen können Fissuren und erosionsartige Defekte im Rahmen einer chronischen Chondropathie vorliegen [12, 58, 60 -65]. Die MRT weist insbesondere bei höhergradigen Knorpelschäden eine hohe diagnostische Genauigkeit auf, während geringgradige Knorpelläsionen dem Nachweis entgehen können [66].

Bei einem Drittel der Luxationspatienten bestehen dislozierte Fragmente als freie Gelenkkörper, die im Befund explizit erwähnt werden sollten, da unter Umständen eine operative Intervention indiziert sein kann. Im Umkehrschluss sollte im Rahmen der Primärdiagnostik bei unklarem Knietrauma mit Nachweis eines Kniegelenksergusses sowie eines intraartikulären Knochenfragments im konventionellen Röntgenbild immer an eine stattgehabte Patellaluxation gedacht werden [67].

Es ist wichtig im MRT-Befund vor allem ostechondrale Fragmente explizit zu erwähnen, da in Abhängigkeit der Lokalisation und der Größe eine Refixation möglich sein kann [68]. Größere und frische osteochondrale Defekte sollten insbesondere bei jüngeren Patienten refixiert werden. Nach Empfehlungen von Rosslen- broich et al. werden Fragmentgrößen ab ca. $1 \mathrm{~cm}^{2}$ als sinnvoll für eine Refixation erachtet, wobei allerdings bisher keine genaueren Daten publiziert wurden, ab welcher Größe eine Fragmentrefixation Erfolg versprechend ist [68]. Rein chondrale Läsionen weisen eine schlechte Heilungstendenz auf $[69,70]$. Auch kleine Fragmente eignen sich nicht zur Refixation, sollten aber entfernt werden, um die Entstehung von freien Gelenkkörpern zu verhindern [68]. Des Weiteren sind knöcherne Ausrisse der medialen Bänder bei erhaltener ligamentärer Struktur von Bedeutung. Diese Verletzungen sind selten, erlauben aber bei umgehender Refixation eine langfristige Wiederherstellung der Primärstabilität, was bei intraligamentären Läsionen deutlich schwieriger ist.

Am lateralen Femurkondylus wird ebenfalls bei fast allen Patienten nach kürzlicher Luxation ein ausgeprägtes Ödem gesehen (॰ Abb.5) [19, 54 - 57]. Hier sind die osteochondralen Verletzungen weniger häufig als an der medialen Patella und werden in der Literatur mit bis $40 \%$ angegeben [71, 72]. Typische Lokalisation ist die zentrale und laterale Hauptbelastungszone [71, 73].

Bei Patienten, die multiple Luxationsereignisse in der Vorgeschichte aufweisen und unter chronischer patellofemoraler Instabilität leiden, liegt häufig eine permanente Subluxation der Patella vor. Dadurch können beträchtliche Schäden der Gelenkflächen bereits beim jungen Patienten verursacht werden, die zu einer frühen patellofemoralen Arthrose führen können, wodurch 


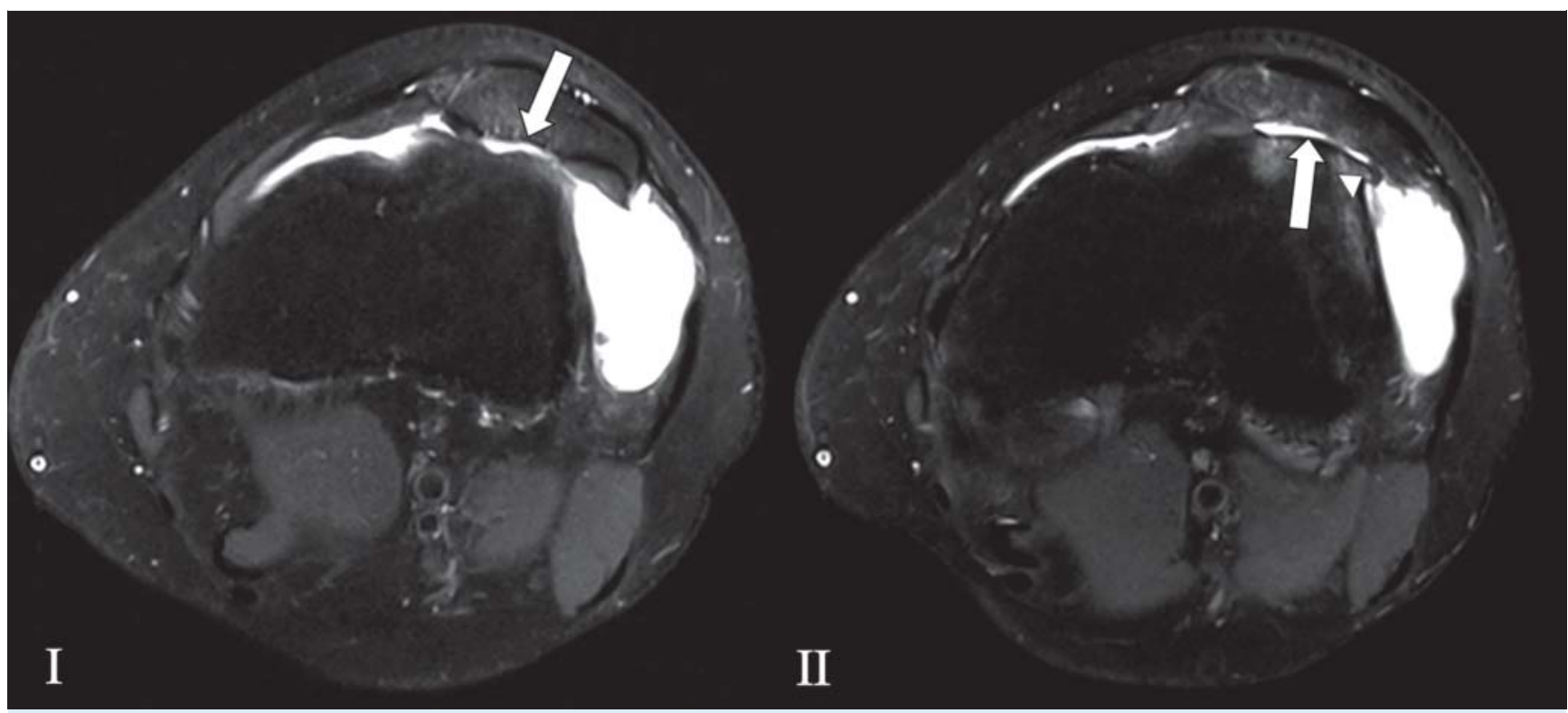

Abb. 8 Knorpelschaden und Arthrose. 28-jährige Patientin mit chronischer patellofemoraler Instabilität nach mehreren Luxationsereignissen. Hochgradiger Knorpeldefekt der zentralen Patella (Pfeil, Bild I) und der la- teralen Trochlea (Pfeil, Bild II). Beginnende patellofemorale Arthrose mit Osteophyten der lateralen Trochleakante (Pfeilspitze, Bild II). die Lebensqualität signifikant eingeschränkt wird [11, 13] (o Abb.8).

\section{Schäden des Kapsel-Band-Apparats}

Für das Verständnis des ligamentären Schadens nach lateraler Patellaluxation ist die Kenntnis des medialen patellofemoralen Ligaments (MPFL) von Bedeutung, da es den wichtigsten passiven Stabilisator darstellt und häufig im Rahmen der Luxation rupturiert. Die medialseitigen Bänder des Patellofemoralgelenks weisen einen komplexen anatomischen Aufbau auf, die im 3Schicht-Modell nach Warren und Mashall zusammengefasst sind [74]: In einer äußeren oberflächlichen Schicht I liegt die Oberschenkelfaszie und die Faszie des M. sartorius. In der mittleren Schicht II sind das fächerförmige MPFL und Faserzüge des medialen Kollateralbands lokalisiert. Die tiefe Schicht III wird durch die eigentliche Gelenkkapsel und weitere Fasern gebildet. Das MPFL ist eine gesonderte horizontal verlaufende Fasergruppe in der mittleren Schicht und gilt als statischer Stabilisator der Patella, da es einem lateralen Abweichen der Kniescheibe entgegenwirkt $[75,76]$. Es verläuft von den superioren zwei Dritteln des medialen Patellarands bis zum femoralen Insertionspunkt anterior des medialen Epicondylus [77, 78] ( $\bullet$ Abb. 7). In den superioren Anteilen ist das Ligament mit der tiefen Faszie des distalen M. vastus medialis verbunden. Am patellaren Ansatz ist das MPFL anterior mit dem medialen Retinakulum verwoben. Das mediale Retinakulum ist eine Verdickung von Faserzügen aus der Schicht I und II, die gemeinsam am medialen Patellarand ansetzen [79]. Im Gegensatz zum MPFL geht das mediale Retinakulum nach dorsal in die Faszie des Vastus medialis obliquus über. In der klinischen Routine sind die Bandstrukturen am besten durch axiale PDw oder T2w FS abgrenzbar, wobei patellarseitig der gemeinsame Ansatz des medialen Retinakulums und MPFL dicker imponiert als die femoralseitige MPFL Insertion ( $\bullet$ Abb. 7) [79 - 81].

Als ligamentärer Stabilisator verhindert das MPFL vor allem ein laterales luxieren der Patella aus der Trochlea. Biomechanische Studien haben gezeigt, dass das MPFL zwischen 50 - 60\% sämtlicher lateralisierender Kräfte der Patella entgegenwirkt [82] und dessen Ruptur signifikante Auswirkungen auf die Entwicklung einer lateralen Instabilität hat [83].

Nach akuter Luxation wird fast immer auch ein Schaden des MPFL nachgewiesen [16, 19, 72, 84-86]. Im Vergleich zur Arthroskopie kann mittels MRT mit höherer Genauigkeit das Vorhandensein, das Ausmaß und der Ort des Schadens spezifiziert werden [87]. Ein Teil der Patienten weist vollständige Rupturen auf, wobei in einigen Fällen auch nur Teilrupturen bestehen können. Im radiologischen Befund sollte die Lokalisation der Ruptur auf das patellare, das mittlere und das femorale Drittel des MPFL spezifiziert werden. Hier ist zu beachten, dass bis zu 50\% der Patienten mehr als eine Rupturstelle des MPFL aufweisen, sodass zum Beispiel beim gleichen Patienten Abrisse sowohl am femoralen als auch am patellaren Ansatz bestehen können [16, 19, 72]. Die Lokalisation der Ruptur sollte im radiologischen Befund dokumentiert werden, da sie entscheidende Bedeutung für die Art der Behandlung hat. Liegt bei Erstluxation beispielsweise eine femoralseitige Läsion vor, ist eine arthroskopische Raffung der medialen Kapsel-Bandstrukturen kontraindiziert, da das geschädigte Gewebe durch einen arthroskopischen Eingriff nicht erreicht und lediglich durch ein offenes Verfahren fixiert werden kann. Liegt eine an mehreren Stellen zu beobachtende ausgeprägte Destruktion des medialen Kapsel-Band-Apparats vor, vor allem in Kombination mit weiteren eine patellofemorale Instabilität begünstigenden Faktoren, ist von einem konservativen Vorgehen abzuraten, da diese keine suffiziente langfristige Wiederherstellung der Stabilität erlauben.

MRT-morphologische Zeichen einer MPFL-Ruptur entsprechen den klassischen Kriterien einer Bandruptur und sind die vollständige Kontinuitätsunterbrechung, der S-förmige Verlauf und das peritendinöse Ödem ( $\bullet$ Abb. 7) [14]. Durch die fehlende mediale Stabilisierung kommt es zur lateralen Subluxation und Verkippung (Tilt) der Patella mit Ausheilung der verletzten Bandstrukturen in dieser Fehlstellung, die dann zu einer Prädisposition zu weiteren Luxationsereignisse führt. Bei der Teilruptur besteht in erster Linie eine fokale Ausdünnung des Bandes und/oder eine fokale T2w-Hyperintensität. 
Insbesondere bei kürzlich stattgehabter Erstluxation liegt ein signifikanter Kniegelenkserguss vor. Je nach Ausmaß des Weichteilschadens und des osteochondralen Defekts können durch intraartikuläres Blut oder Fettmark Flüssigkeitsspiegel entstehen. Patienten mit rezidivierenden Luxationen oder chronischer patellofemoraler Instabilität weisen gelegentlich keinen signifikanten Erguss auf, da bei erneuter Luxation die bereits insuffizienten Weichteile nicht mehr beschädigt werden. Häufig wird nach akuter Luxation auch ein Ödem und eine Zerrung des M. vastus medialis obliquus beobachtet [19, 72], der vor allem in Flexion ebenfalls als medialer Stabilisator wirkt und bei lateraler Luxation durch seinen Ansatz an der Patella neben dem MPFL ebenfalls signifikanten Zugkräften ausgesetzt ist. In selteneren Fällen können durch die enge anatomische Lagebeziehung Schäden des medialen Kollateralbands und am medialen Meniskus bestehen, die klinisch ein ähnliches Beschwerdebild wie die spontan reponierte Patellaluxation aufweisen können, weswegen es sinnvoll ist, im Befund explizit auf diese Strukturen einzugehen.

\section{Therapie \\ $\nabla$}

Das therapeutische Spektrum der Patellaluxation reicht von konservativer Therapie über operative Standardverfahren wie Raffung des medialen Kapsel-Band-Apparats, Naht/Augmentation des MPFL und laterale Retinakulumspaltung (lateral release) bis hin zu Osteotomien der Trochlea (Trochleaplastik), der Tuberositas tibiae (Medialisierung, Anteromedialisierung) oder des Femurs/der Tibia (Derotation) [49, 88]. Ausschlaggebend für das Behandlungskonzept sind die Häufigkeit der stattgehabten Luxation, das Verletzungsmuster und der vermutete Pathomechanismus.

Bei Erstluxation der Patella war bis dato eine primär konservativ Behandlung vorgesehen, wenn keine größeren osteochondralen Verletzungen festzustellen waren. Allerdings haben mehrere Studien gezeigt, dass nach Erstluxation Reluxationsraten zwischen $15-35 \%$ bestehen [ 89 - 92]. Neu entwickelte Operationstechniken sprechen für eine primär operative Versorgung der Erstluxation durch Rekonstruktion des medialen patellofemoralen Ligaments [93].

Übereinstimmung besteht bei der operativen Therapie der rezidivierenden Patellaluxation. Rezidivierende Luxationen werden in Abhängigkeit des zugrunde liegenden individuellen Risikoprofils behandelt [43, 94, 95], weswegen die MRT-Diagnostik einen wichtigen Stellenwert in der Therapieplanung einnimmt. Weisen diese Patienten im einfachsten Fall lediglich eine Trochleadysplasie Grad B und keine Patella alta und keinen pathologischen TTTG auf, so ist die klassische mediale Raffung in arthroskopischer oder offener Technik Verfahren der Wahl [96, 97]. Bei Patienten mit einer Trochleadysplasie Typ B oder C und deutlicher Subluxationsstellung ist die Rekonstruktion des medialen patellofemoralen Ligaments zu empfehlen [98]. Dieses Verfahren sollte im Falle eines pathologischen TT-TG und/oder einer Patella alta mit einem Versatz der Tuberositas tibiae kombiniert werden [99]. Bei höhergradigen Dysplasien der Trochlea vom Typ C und D sind Trochleaplastiken zur Rekonstruktion des trochleären Sulkus allein oder in Kombination mit medial stabilisierenden Verfahren (MPFL-Rekonstruktion, mediale Raffung) indiziert. In den seltenen Fällen, in denen Rotationsdeformitäten des Femurs oder der Tibia die ausschlaggebende Pathologie der patellofemoralen Instabilität darstellen, können Derotationsosteotomien isoliert oder in Kombination mit weiteren Verfahren zur Anwendung kommen.
Es existiert noch eine inzwischen fast unüberschaubare Anzahl an weiteren Techniken oder Modifikation von oben beschriebenen Verfahren. Jedoch gilt auch hier die Prämisse, dass das Verständnis der Ursache der patellofemoralen Instabilität die Wahl des entsprechenden Verfahrens bestimmt.

Weitere zusätzliche Informationen und ausführliches Bildmaterial über das Thema sind in einem Radiographics Artikel verfügbar: PMID: 20631363 [14].

\section{Literatur}

1 Oestern S, Varoga D, Lippross $S$ et al. Patellaluxation. Unfallchirurg 2011; 114: $345-358$

2 Wirth T. Patellaluxationen. Unfallchirurg 2011; 114: 388 -395

3 Petersen $W$, Forkel $P$, Achtnich A. Chronische patellofemorale Instabilität. Unfallchirurg 2012; 115: 397-409

4 Atkin DM, Fithian DC, Marangi KS et al. Characteristics of patients with primary acute lateral patellar dislocation and their recovery within the first 6 months of injury. Am J Sports Med 2000; 28: 472-479

5 Fithian DC, Paxton EW, Stone ML et al. Epidemiology and natural history of acute patellar dislocation. Am J Sports Med 2004; 32: 1114-1121

6 Sillanpaa P, Mattila VM, Iivonen T et al. Incidence and risk factors of acute traumatic primary patellar dislocation. Med Sci Sports Exerc 2008; 40: 606-611

7 Hsiao M, Owens BD, Burks R et al. Incidence of acute traumatic patellar dislocation among active-duty United States military service members. Am J Sports Med 38: 1997-2004

8 Stefancin JJ, Parker RD. First-time traumatic patellar dislocation: a systematic review. Clin Orthop Relat Res 2007; 455: $93-101$

9 Hawkins RJ, Bell RH, Anisette G. Acute patellar dislocations. The natural history. Am J Sports Med 1986; 14: 117-120

10 Maenpaa $H$, Huhtala $H$, Lehto MU. Recurrence after patellar dislocation. Redislocation in 37/75 patients followed for 6-24 years. Acta Orthop Scand 1997; 68: 424-426

11 Maenpaa H, Lehto MU. Patellofemoral osteoarthritis after patellar dislocation. Clin Orthop Relat Res 1997; 339: 156-162

12 Nomura E, Inoue M. Second-look arthroscopy of cartilage changes of the patellofemoral joint, especially the patella, following acute and recurrent patellar dislocation. Osteoarthritis Cartilage 2005; 13: 10291036

13 Sillanpaa PJ, Mattila VM, Visuri T et al. Patellofemoral osteoarthritis in patients with operative treatment for patellar dislocation: a magnetic resonance-based analysis. Knee Surg Sports Traumatol Arthrosc 2010; 19: $230-235$

14 Diederichs G, Issever AS, Scheffler S. MR imaging of patellar instability: injury patterns and assessment of risk factors. Radiographics 2010; 30 : 961-981

15 Kohlitz T, Scheffler S, Jung T et al. Prevalence and patterns of anatomical risk factors in patients after patellar dislocation: a case control study using MRI. Eur Radiol; Epub 2012 Nov 29

16 Balcarek P, Ammon J, Frosch S et al. Magnetic resonance imaging characteristics of the medial patellofemoral ligament lesion in acute lateral patellar dislocations considering trochlear dysplasia, patella alta, and tibial tuberosity-trochlear groove distance. Arthroscopy 2010; 26: $926-935$

17 Carrillon Y, Abidi H, Dejour D et al. Patellar instability: assessment on MR images by measuring the lateral trochlear inclination-initial experience. Radiology 2000; 216: 582-585

18 Dejour H, Walch G, Nove-Josserand L et al. Factors of patellar instability: an anatomic radiographic study. Knee Surg Sports Traumatol Arthrosc 1994; $2: 19-26$

19 Elias DA, White LM, Fithian DC. Acute lateral patellar dislocation at MR imaging: injury patterns of medial patellar soft-tissue restraints and osteochondral injuries of the inferomedial patella. Radiology 2002; 225: $736-743$

20 Escala JS, Mellado JM, Olona $M$ et al. Objective patellar instability: MRbased quantitative assessment of potentially associated anatomical features. Knee Surg Sports Traumatol Arthrosc 2006; 14: 264-272

21 Pfirrmann CW, Zanetti $M$, Romero J et al. Femoral trochlear dysplasia: MR findings. Radiology 2000; 216: 858-864

22 Weber-Spickschen TS, Spang J, Kohn L et al. The relationship between trochlear dysplasia and medial patellofemoral ligament rupture loca- 
tion after patellar dislocation: an MRI evaluation. Knee 2010; 18: 185 188

23 Dejour H, Walch G, Neyret P et al. Dysplasia of the femoral trochlea. Rev Chir Orthop Reparatrice Appar Mot 1990; 76: 45-54

24 Salzmann GM, Weber TS, Spang JT et al. Comparison of native axial radiographs with axial MR imaging for determination of the trochlear morphology in patients with trochlear dysplasia. Arch Orthop Trauma Surg 2010; 130: 335 - 340

25 Balcarek P, Jung K, Ammon J et al. Anatomy of lateral patellar instability: trochlear dysplasia and tibial tubercle-trochlear groove distance is more pronounced in women who dislocate the patella. Am J Sports Med 2010; 38: 2320-2327

26 Balcarek P, Walde TA, Frosch $S$ et al. Patellar dislocations in children, adolescents and adults: a comparative MRI study of medial patellofemoral ligament injury patterns and trochlear groove anatomy. Eur J Radiol 2010; 79: 415-420

27 Barnett AJ, Prentice M, Mandalia V et al. Patellar height measurement in trochlear dysplasia. Knee Surg Sports Traumatol Arthrosc 2009; 17: $1412-1415$

28 Biedert RM, Albrecht S. The patellotrochlear index: a new index for assessing patellar height. Knee Surg Sports Traumatol Arthrosc 2006; 14: 707-712

29 Neyret $P$, Robinson AH, Le Coultre B et al. Patellar tendon length - the factor in patellar instability? Knee 2002; 9: 3-6

30 Ward SR, Terk MR, Powers CM. Patella alta: association with patellofemoral alignment and changes in contact area during weight-bearing. J Bone Joint Surg Am 2007; 89: 1749-1755

31 Feller JA, Amis AA, Andrish JT et al. Surgical biomechanics of the patellofemoral joint. Arthroscopy 2007; 23: $542-553$

32 Barnett AJ, Prentice M, Mandalia V et al. Patellar height measurement in trochlear dysplasia. Knee Surg Sports Traumatol Arthrosc 2009; 17: $1412-1415$

33 Seil R, Muller B, Georg T et al. Reliability and interobserver variability in radiological patellar height ratios. Knee Surg Sports Traumatol Arthrosc 2000; 8: $231-236$

34 Miller TT, Staron RB, Feldman F. Patellar height on sagittal MR imaging of the knee. Am J Roentgenol 1996; 167: 339-341

35 Insall J, Salvati E. Patella position in the normal knee joint. Radiology 1971; 101: $101-104$

36 Insall J, Goldberg $V$, Salvati E. Recurrent dislocation and the high-riding patella. Clin Orthop Relat Res 1972; 88: 67-69

37 Caton J. Method of measuring the height of the patella. Acta Orthop Belg 1989; 55: $385-386$

38 Thevenin-Lemoine C, Ferrand M, Courvoisier A et al. Is the CatonDeschamps index a valuable ratio to investigate patellar height in children? J Bone Joint Surg Am 2011; 93: e35

39 Balcarek P, Jung K, Frosch KH et al. Value of the tibial tuberosity-trochlear groove distance in patellar instability in the young athlete. Am J Sports Med 2011; 39: 1756-1761

40 Tsujimoto K, Kurosaka M, Yoshiya S et al. Radiographic and computed tomographic analysis of the position of the tibial tubercle in recurrent dislocation and subluxation of the patella. Am J Knee Surg 2000; 13: $83-88$

41 Schoettle PB, Zanetti M, Seifert B et al. The tibial tuberosity-trochlear groove distance; a comparative study between CT and MRI scanning. Knee 2006; 13: 26-31

42 Pandit S, Frampton C, Stoddart J et al. Magnetic resonance imaging assessment of tibial tuberosity-trochlear groove distance: normal values for males and females. Int Orthop 2011; 35: 1799-1803

43 Colvin AC, West RV. Patellar instability. J Bone Joint Surg Am 2008; 90 : $2751-2762$

44 Diederichs G, Koehlitz T, Kornaropoulos E et al. MRI Analysis of Rotational Alignment in Patients with Patellar Dislocation. Am J Sports Med 2013; 41: $51-57$

45 Cameron JC, Saha S. External tibial torsion: an underrecognized cause of recurrent patellar dislocation. Clin Orthop Relat Res 1996; 328 : $177-184$

46 Cooke TD, Price N, Fisher B et al. The inwardly pointing knee. An unrecognized problem of external rotational malalignment. Clin Orthop Relat Res 1990; 260: 56-60

47 Turner MS, Smillie IS. The effect of tibial torsion of the pathology of the knee. J Bone Joint Surg Br 1981, 63-B: 396-398

48 Reikeras 0 . Patellofemoral characteristics in patients with increased femoral anteversion. Skeletal Radiol 1992; 21: 311 - 313
49 Frosch S, Balcarek P, Walde TA et al. Die Therapie der Patellaluxation: Eine systematische Literaturanalyse. Z Orthop Unfall 2011; 149: 630-645

50 Arendt EA, Fithian DC, Cohen E. Current concepts of lateral patella dislocation. Clin Sports Med 2002; 21: 499-519

51 Stanitski CL. Articular hypermobility and chondral injury in patients with acute patellar dislocation. Am J Sports Med 1995; 23: 146-150

52 Runow A. The dislocating patella. Etiology and prognosis in relation to generalized joint laxity and anatomy of the patellar articulation. Acta Orthop Scand Suppl 1983; 201: 1-53

53 Nomura E, Inoue M, Kobayashi S. Generalized joint laxity and contralateral patellar hypermobility in unilateral recurrent patellar dislocators. Arthroscopy 2006; 22: $861-865$

54 Kirsch MD, Fitzgerald SW, Friedman $H$ et al. Transient lateral patellar dislocation: diagnosis with MR imaging. Am J Roentgenol 1993; 161 : $109-113$

55 Lance E, Deutsch AL, Mink JH. Prior lateral patellar dislocation: MR imaging findings. Radiology 1993; 189: $905-907$

56 Spritzer CE, Courneya DL, Burk DL Jr et al. Medial retinacular complex injury in acute patellar dislocation: MR findings and surgical implications. Am J Roentgenol 1997; 168: 117-122

57 Virolainen $H$, Visuri T, Kuusela T. Acute dislocation of the patella: MR findings. Radiology 1993; 189: $243-246$

58 Vollnberg B, Koehlitz T, Jung T et al. Prevalence of cartilage lesions and early osteoarthritis in patients with patellar dislocation. Eur Radiol 2012; 22: 2347-2356

59 Jerabek SA, Asnis PD, Bredella MA et al. Medial patellar ossification after patellar instability: a radiographic finding indicative of prior patella subluxation/dislocation. Skeletal Radiol 2009; 38: 785-790

60 Nomura E, Inoue M. Cartilage lesions of the patella in recurrent patellar dislocation. Am J Sports Med 2004; 32: 498-502

61 Nomura E, Inoue $M$, Kurimura $M$. Chondral and osteochondral injuries associated with acute patellar dislocation. Arthroscopy 2003; 19: $717-721$

62 Vollnberg B, Koehlitz T, Jung $T$ et al. Prevalence of cartilage lesions and early osteoarthritis in patients with patellar dislocation. Eur Radiol 2012; 22: 2347-2356

63 Arnoldi AP, Weckbach S, Nussbickel C et al. MRT-basierte Knorpelvolumetrie nach Kreuzbandersatzplastik in Korrelation mit qualitativen Gelenkveränderungen und dem klinischen Outcome. Gibt es Hinweise auf frühzeitige posttraumatische degenerative Veränderungen? Fortschr Röntgenstr 2011; 183: 1138-1144

64 Horng A, Raya J, Zscharn M et al. Lokoregionale Belastungszonen des Gelenkknorpels im gesamten Knie nach Belastung in unterschiedlichen Flexionsgraden mit 3D-MR-Volumetrie bei 3T. Fortschr Röntgenstr 2011; 183: $432-440$

65 Korn A, Horger M, Albrecht D et al. Korbhenkelrisse. Fortschr Röntgenstr 2011; 183: 91 - 94

66 von Engelhardt LV, Raddatz M, Bouillon $B$ et al. How reliable is MRI in diagnosing cartilaginous lesions in patients with first and recurrent lateral patellar dislocations? BMC Musculoskelet Disord 2010; 11: 149

67 Haas JP, Collins MS, Stuart MJ. The "sliver sign": a specific radiographic sign of acute lateral patellar dislocation. Skeletal Radiol 2011; 41: $595-601$

68 Rosslenbroich S, Raschke M, Petersen W. Akute osteochondrale Läsionen nach Patellaluxation. Unfallchirurg 2012; 115: 392-396

69 Mankin HJ. The reaction of articular cartilage to injury and osteoarthritis (second of two parts). N Engl J Med 1974; 291: 1335-1340

70 Mankin HJ. The reaction of articular cartilage to injury and osteoarthritis (first of two parts). N Engl J Med 1974; 291: 1285-1292

71 Sanders TG, Paruchuri NB, Zlatkin MB. MRI of osteochondral defects of the lateral femoral condyle: incidence and pattern of injury after transient lateral dislocation of the patella. Am J Roentgenol 2006; 187: $1332-1337$

72 Seeley M, Bowman KF, Walsh $C$ et al. Magnetic resonance imaging of acute patellar dislocation in children: patterns of injury and risk factors for recurrence. J Pediatr Orthop 2012; 32: 145 - 155

73 Mashoof AA, Scholl MD, Lahav A et al. Osteochondral injury to the midlateral weight-bearing portion of the lateral femoral condyle associated with patella dislocation. Arthroscopy 2005; 21: 228-232

74 Warren LF, Marshall JL. The supporting structures and layers on the medial side of the knee: an anatomical analysis. J Bone Joint Surg Am 1979; 61: 56-62 
75 Hautamaa PV, Fithian DC, Kaufman KR et al. Medial soft tissue restraints in lateral patellar instability and repair. Clin Orthop Relat Res 1998; 349: $174-182$

76 Stoller DW, Li AE, Anderson LJ et al. The Knee. In: Stoller DW (ed) Magnetic Resonance Imaging in Orthopedics and Sports Medicine Third Edition. Baltimore: Lippincott Williams \& Wilkins; 2007, 605-614

77 Tuxoe JI, Teir M, Winge $S$ et al. The medial patellofemoral ligament: a dissection study. Knee Surg Sports Traumatol Arthrosc 2002; 10: $138-140$

78 Feller JA, Feagin JA Jr, Garrett WE et al. The medial patellofemoral ligament revisited: an anatomical study. Knee Surg Sports Traumatol Arthrosc 1993; 1: $184-186$

79 Ruiz ME, Erickson SJ. Medial and lateral supporting structures of the knee. Normal MR imaging anatomy and pathologic findings. Magn Reson Imaging Clin N Am 1994; 2: 381 - 399

80 Thawait SK, Soldatos T, Thawait GK et al. High resolution magnetic resonance imaging of the patellar retinaculum: normal anatomy, common injury patterns, and pathologies. Skeletal Radiol 2012; 41: 137 148

81 De Maeseneer M, Van Roy F, Lenchik L et al. Three layers of the medial capsular and supporting structures of the knee: MR imaging-anatomic correlation. Radiographics 2000; 20: 83-89

82 Amis AA, Firer P, Mountney J et al. Anatomy and biomechanics of the medial patellofemoral ligament. Knee 2003; 10: 215-220

83 Senavongse $W$, Amis AA. The effects of articular, retinacular, or muscular deficiencies on patellofemoral joint stability. J Bone Joint Surg $\mathrm{Br}$ 2005; 87: $577-582$

84 Guerrero $P$, Li X, Patel $\mathrm{K}$ et al. Medial patellofemoral ligament injury patterns and associated pathology in lateral patella dislocation: an MRI study. Sports Med Arthrosc Rehabil Ther Technol 2009; 1: 17

85 Sanders TG, Morrison WB, Singleton BA et al. Medial patellofemoral ligament injury following acute transient dislocation of the patella: MR findings with surgical correlation in 14 patients. J Comput Assist Tomogr 2001; 25: 957-962

86 Sillanpaa PJ, Peltola E, Mattila VM et al. Femoral Avulsion of the Medial Patellofemoral Ligament After Primary Traumatic Patellar Dislocation Predicts Subsequent Instability in Men: A Mean 7-Year Nonoperative Follow-Up Study. Am J Sports Med 2009; 37: 1513-1521
87 Balcarek $P$, Walde TA, Frosch $S$ et al. MRI but not arthroscopy accurately diagnoses femoral MPFL injury in first-time patellar dislocations. Knee Surg Sports Traumatol Arthrosc 2012; 20: 1575 -1580

88 Redziniak DE, Diduch DR, Mihalko WM et al. Patellar instability. J Bone Joint Surg Am 2009; 91: 2264-2275

89 Christiansen SE, Jakobsen BW, Lund B et al. Isolated repair of the medial patellofemoral ligament in primary dislocation of the patella: a prospective randomized study. Arthroscopy 2008; 24: $881-887$

90 Nikku R, Nietosvaara Y, Aalto $K$ et al. Operative treatment of primary patellar dislocation does not improve medium-term outcome: A 7 year follow-up report and risk analysis of 127 randomized patients. Acta Orthop 2005; 76: 699-704

91 Nikku R, Nietosvaara Y, Kallio PE et al. Operative versus closed treatment of primary dislocation of the patella. Similar 2-year results in 125 randomized patients. Acta Orthop Scand 1997; 68: 419-423

92 Sillanpaa PJ, Mattila VM, Maenpaa $\mathrm{H}$ et al. Treatment with and without initial stabilizing surgery for primary traumatic patellar dislocation. A prospective randomized study. J Bone Joint Surg Am 2009; 91: 263 273

93 Bitar AC, Demange MK, D'Elia CO et al. Traumatic patellar dislocation: nonoperative treatment compared with MPFL reconstruction using patellar tendon. Am J Sports Med 2011; 40: 114-122

94 Mulford JS, Wakeley CJ, Eldridge JD. Assessment and management of chronic patellofemoral instability. J Bone Joint Surg $\mathrm{Br} 2007$; 89: $709-716$

95 White BJ, Sherman OH. Patellofemoral instability. Bull NYU Hosp Jt Dis 2009; 67: $22-29$

96 Nam EK, Karzel RP. Mini-open medial reefing and arthroscopic lateral release for the treatment of recurrent patellar dislocation: a mediumterm follow-up. Am J Sports Med 2005; 33: 220-230

97 Rillmann P, Fischer A, Berbig R et al. Arthroskopisch unterstützte mediale Retinakulumnaht nach akuter Erstluxation der Patella. Unfallchirurg 1999; 102: 167-172

98 LeGrand $A B$, Greis PE, Dobbs RE et al. MPFL reconstruction. Sports Med Arthrosc 2007; 15: 72 - 77

99 Caton JH, Dejour D. Tibial tubercle osteotomy in patello-femoral instability and in patellar height abnormality. Int Orthop 2010; 34: $305-$ 309 